\title{
Noninertial effects on a scalar field in a spacetime with a magnetic screw dislocation
}

\author{
Ricardo L. L. Vitória ${ }^{a}$ \\ Departamento de Física e Química, Universidade Federal do Espírito Santo, Av. Fernando Ferrari, 514, Goiabeiras, Vitória, ES 29060-900, Brazil
}

Received: 13 March 2019 / Accepted: 30 September 2019 / Published online: 13 October 2019

(C) The Author(s) 2019

\begin{abstract}
We investigate rotating effects on a charged scalar field immersed in spacetime with a magnetic screw dislocation. In addition to the hard-wall potential, which we impose to satisfy a boundary condition from the rotating effect, we insert a Coulomb-type potential and the KleinGordon oscillator into this system, where, analytically, we obtain solutions of bound states which are influenced not only by the spacetime topology, but also by the rotating effects, as a Sagnac-type effect modified by the presence of the magnetic screw dislocation.
\end{abstract}

\section{Introduction}

In the context of condensed matter physics, Katanaev and Volovich [1] formulated a description of defects in a threedimensional continuous elastic solid medium, where such defects may be associated with curvature or torsion of the continuous medium. Then, Puntigam and Soleng [2] went further with this formulation through the generalization of the Volterra distortions, considering the temporal coordinate, that is, $(3+1)$ dimensions, in order to adapt these defects in a Einstein-Cartan gravity and introduce the concept of distorted spacetimes. According to this formulation, disclinations are associated with curvature, while dislocations are associated with torsion. In particular, the dislocations can be typified as spiral and screw [3]. Effects associated with the topology of a medium with dislocations have been investigated in crystal structures through differential geometry [4].

Recently, dislocations have been investigated in nonrelativistic and relativistic quantum systems. The spiral dislocation, in the nonrelativistic context, has been investigated in the harmonic oscillator [5]; in the relativistic context, it has been investigated in a scalar field in a noninertial frame [6]. The screw dislocation, in the nonrelativistic context, has been applied in the harmonic oscillator [7,8], in the Landau quan-

a e-mails: ricardo.vitoria@ufes.br; ricardo.vitoria@pq.cnpq.br tization [9-11], in the doubly anharmonic oscillator [12], in a Landau quantization for an induced electric dipole [13] and in noninertial effects on a nonrelativistic Dirac particle [14]; in the relativistic context, it has been studied in the Dirac oscillator [15,16], in the Klein-Gordon oscillator [17-19] and in an analogue of the AharonovBohm effect for bound states in a position-dependent mass system [20]. The metric that characterizes the space-time with a screw dislocation is defined as follows $(c=\hbar=1)$ [2]:

$d s^{2}=-d t^{2}+d \rho^{2}+\rho^{2} d \varphi^{2}+(d z+\chi d \varphi)^{2}$,

where $\rho=\sqrt{x^{2}+y^{2}}$ is the radial coordinate and $\chi$ is the parameter associated to the screw dislocation, that, in condensed matter physics, this parameter is related to the Burgers vector $\mathbf{b}$ via $\chi=\frac{b}{2 \pi}[1-4]$. It is important to mention that the screw dislocation (torsion) corresponds to a singularity at the origin [7]. In addition, the spatial part of the metric (1) is called in the literature as Katanaev and Volovich line element [2].

In an analysis by Landau and Lifshitz on the effects of rotation in the Minkowski spacetime with cylindrical symmetry, they showed that the radial coordinate becomes restricted in an interval, where this restriction is an effect directly related to the uniform rotation [21]. This restriction from the effects of uniform rotation has been widely used for studies in a relativistic quantum mechanics system, for example, in a Dirac particle [22], in a relativistic Landau-He-McKellar-Wilkens quantization [23], on the Dirac oscillator [24], on a scalar field in the spacetime with space-like dislocation and in the spacetime with a spiral dislocation [6], on the quantum dynamics of scalar bosons [25], in the relativistic quantum motion of spin-0 particles under in the cosmic string spacetime [26], in the Duffin-Kemmer-Petiau equation with magnetic cosmic string background [27]. In the nonrelativistic case, this restriction has been studied in a Dirac particle in the spacetime with a screw dislocation [16] and on nonrelativistic topological quantum scattering [28]. However, a point that has 
not been analyzed in the literature is the rotating effect on the scalar field by considering the spacetime with a magnetic screw dislocation as background, that is, the screw dislocation has in the core a magnetic field with magnetic quantum flux $\Phi_{B}$ and outside the topological defect this magnetic field vanishes [7,11].

To introduce a uniform rotation in the spacetime with a screw dislocation, consider the transformation $\varphi \rightarrow \varphi+w t$ into the Eq. (1), where $w$ is the constant angular frequency of the rotating frame, which gives us the metric

$$
\begin{aligned}
d s^{2}= & -\left(1-\rho^{2} w^{2}-\chi^{2} w^{2}\right) d t^{2}+d \rho^{2} \\
& +\left(\rho^{2}+\chi^{2}\right) d \varphi^{2}+2\left(\rho^{2}+\chi^{2}\right) w d \varphi d t \\
& +2 \chi d \varphi d z+2 \chi w d t d z+d z^{2} .
\end{aligned}
$$

In order to make the component $g_{00}$ remain negative, we extract the information that the radial coordinate becomes restricted by consequence of the rotating frame:

$0 \leq \rho<\frac{\sqrt{1-\chi^{2} w^{2}}}{w}$.

We can note that, in addition to the angular frequency of the noninertial frame, the inequality (3) is determined by the parameter related to the topological defect $\chi$. We can also note that, for $\rho>\frac{\sqrt{1-\chi^{2} w^{2}}}{w}$ we would have a particle is placed outside of the light-cone $[6,14]$. By taking $\chi=0$ we recover the result obtained by Landau and Lifshitz [21].

In this paper, we investigate the relativistic AharonovBohm effect for bound states $[29,30]$ on a scalar field in a spacetime with a screw dislocation, where this field is subject to confinement potentials in a uniformly rotating frame. We begin our analysis with the hard-wall potential. After, we consider a scalar field with position-dependent mass interacting with a Coulomb-type central potential. And, finally, we inserted the Klein-Gordon oscillator [31] and investigated the harmonic effects coming from this model of relativistic oscillator. In all these cases, we obtain analytical solutions, where they are not only influenced by the topology of the spacetime, but also the effects of rotation.

For an electrically charged scalar field that interacts with the electromagnetic field in a curved spacetime or a spacetime with torsion, the KleinGordon equation is written in the form $[6,18,32]$ :

$\frac{1}{\sqrt{-g}}\left(\partial_{\mu}-i q A_{\mu}\right)\left(\sqrt{-g} g^{\mu \nu}\right)\left(\partial_{\nu}-i q A_{\nu}\right) \phi-m^{2} \phi=0$,

where $g=\operatorname{det}\left(g_{\mu \nu}\right)$, that, for the Eq. (2), we have $g=-\rho^{2}$, $g^{\mu \nu}$ is inverse metric tensor, $m$ is the rest mass of the scalar field and $A_{\mu}=\left(0,0, A_{\varphi}, 0\right)$ is the electromagnetic 4-vector potential, where $A_{\varphi}$ is given by $[18,33,34]$

$A_{\varphi}=\frac{\Phi_{B}}{2 \pi}$, where $\Phi_{B}=$ const. is the Aharonov-Bohm quantum flux through the core of the topological defect [7,11]. It is noteworthy that the Aharonov-Bohm effect has been investigated in several branches of physics, for example, in graphene [35], in a neo-Newtonian theory [36], in bound states of massive fermions [37], in scattering of dislocated wave fronts [38], in torsion effects on a relativistic position-dependent mass system [20], in Kaluza-Klein theory [39,40] and with a nonminimal Lorentz-violating coupling [41]. In this way, from the Eqs. (2) and (5), the Klein-Gordon equation (4) becomes

$$
\begin{gathered}
-\frac{\partial^{2} \phi}{\partial t^{2}}+2 w \frac{\partial^{2} \phi}{\partial t \partial \varphi}-i \frac{q w \Phi_{B}}{\pi} \frac{\partial \phi}{\partial t}+\frac{1}{\rho^{2}} \frac{\partial^{2} \phi}{\partial \varphi^{2}} \\
-i \frac{q \Phi_{B}}{\pi \rho^{2}} \frac{\partial \phi}{\partial \varphi}-\frac{q^{2} \Phi_{B}^{2}}{4 \pi^{2} \rho^{2}} \phi-w^{2} \frac{\partial^{2} \phi}{\partial \varphi^{2}}+i \frac{q \Phi_{B} w^{2}}{\pi} \frac{\partial \phi}{\partial \varphi} \\
-\frac{q^{2} \Phi_{B}^{2} w^{2}}{4 \pi^{2}} \phi-\frac{2 \chi}{\rho^{2}} \frac{\partial^{2} \phi}{\partial \varphi \partial z}+i \frac{q \chi \Phi_{B}}{\pi \rho^{2}} \frac{\partial \phi}{\partial z}+\frac{\partial^{2} \phi}{\partial z^{2}} \\
+\frac{\chi^{2}}{\rho^{2}} \frac{\partial^{2} \phi}{\partial z^{2}}+\frac{\partial^{2} \phi}{\partial \rho^{2}}+\frac{1}{\rho} \frac{\partial \phi}{\partial \rho}-m^{2} \phi=0,
\end{gathered}
$$

which describes an electrically charged scalar field in the spacetime with a magnetic screw dislocation in a uniformly rotating frame.

The structure of this paper is as follows: in the Sect. 2, for a particular case, we investigate the effects of the spacetime topology and of rotation on an electrically charged scalar field subject to the hard-wall potential, where it is possible to obtain the energy levels of this system; in the Sect.3, we inserted a Coulomb-type potential in the Klein-Gordon equation via the mass term and, for a particular case, extracted the energy profile of this system; in the Sect.4, through a nonminimal coupling in the Klein-Gordon equation, we insert a relativistic oscillator model and analyze the harmonic effects on the scalar field in a uniformly rotating frame in the spacetime with a magnetic screw dislocation, where we determine two energy profiles for the system; in the Sect. 5, we present our conclusions.

\section{Hard-wall confining potential}

In this section, we use the restriction in the radial coordinate, which is a consequence of the rotating effects, to introduce the hard-wall potential into the system. This type of potential confinement has been studied in a Landau-Aharonov-Casher system in the cosmic string spacetime [42], in a Landau-type quantization from a Lorentz symmetry violation background [43], in persistent currents for a moving neutral particle with a permanent electric dipole moment [44] and on a Dirac neutral particle in analogous way to a quantum dot [45]. Then, let us return to the Eq. (6). The solution to Eq. (6) is given as 
follows

$\phi(\rho, \varphi, z, t)=e^{-i(\mathcal{E} t-l \varphi-k z)} R(\rho)$,

where $l=0, \pm 1, \pm 2, \ldots$ is eigenvalue of angular momentum operator $\hat{L}_{z}=-i \partial_{\varphi}$ and $-\infty<z<\infty$ is eigenvalue of the linear momentum operator $\hat{p}_{z}=-i \partial_{z}$, where both commute with the hamiltonian. Then, by substituting the Eq. (7) into the Eq. (6), we obtain the radial differential equation for the radial wave function $R(\rho)$ :

$\frac{d^{2} R}{d \rho^{2}}+\frac{1}{\rho} \frac{d R}{d \rho}-\frac{\iota^{2}}{\rho^{2}} R+\alpha^{2} R=0$,

where

$$
\begin{aligned}
\alpha^{2} & =\left[\mathcal{E}+w\left(l-\frac{q \Phi_{B}}{2 \pi}\right)\right]^{2}-m^{2}-k^{2} ; \\
\iota^{2} & =\left(l-k \chi-\frac{q \Phi_{B}}{2 \pi}\right)^{2} .
\end{aligned}
$$

The Eq. (8) is the Bessel equation [46] and its general solution is given as follows

$R(\rho)=C_{1} J_{|\iota|}(\alpha \rho)+C_{2} N_{|\iota|}(\alpha \rho)$,

with $C_{1}$ and $C_{2}$ being constants, $J_{|\ell|}$ the Bessel function of first kind and $N_{|\iota|}$ the Neumann function [46]. It is important to note that the Neumann function, or Bessel function of second kind, diverges at $\rho \rightarrow 0$. However, as we are interested in well-behaved solutions, it is necessary that $C_{2}=0$, by making the Eq. (10) be rewritten as:

$R(\rho)=C_{1} J_{|\iota|}(\alpha \rho)$.

As seen earlier, in Eq. (3), the radial coordinate is limited due to the rotating effects. Thus, here we will impose that the radial wave function $R(\rho)$ vanishes according to the boundary condition

$\lim _{\rho \rightarrow \rho_{0}} R(\rho)=0$

where $\rho_{0}=\frac{\sqrt{1-\chi^{2} w^{2}}}{w}$. From the mathematical point of view, this boundary condition is known in the literature as the Dirichlet boundary condition; from the physical point of view, the radial wave function is in a region where the hardwall potential is present at the point $\rho_{0}$. To give continuity, let us consider a particular case of asymptotic expansion of the Bessel function of first kind, that is, let us consider $\alpha \rho_{0} \gg 1$. In this particular case, we can write $J_{|\iota|}$ in the form [46,47]

$J_{|\iota|}\left(\alpha \rho_{0}\right) \propto \cos \left(\alpha \rho_{0}-\frac{\pi|\iota|}{2}-\frac{\pi}{4}\right)$.

Therefore, by substituting the Eq. (13) into the Eq. (11), and then into the Eq. (12), we obtain

$$
\begin{aligned}
\mathcal{E}_{k, l, n} \approx & -w\left(l-\frac{q \Phi_{B}}{2 \pi}\right) \\
& \pm \sqrt{m^{2}+k^{2}+\frac{\pi w^{2}}{\left(1-\chi^{2} w^{2}\right)}\left(n+\frac{1}{2}\left|l-k \chi-\frac{q \Phi_{B}}{2 \pi}\right|+\frac{3}{4}\right)^{2}}
\end{aligned}
$$

where $n=0,1,2,3, \ldots$ The Eq. (14) represents the relativistic energy levels of a charged scalar field under the hard-wall potential effects induced for rotation in a spacetime with a magnetic screw dislocation. We can note that the spacetime topology influences the relativistic energy levels through the presence of the parameter associated with the topological defect. This influence can be seen through the change on the eigenvalues of the angular momentum by providing an effective angular momentum $l_{\text {eff }}=l-k \chi$, that is, a analogous effect to the Aharonov-Bohm effect for bound states [30] and in the definition of the fixed radius $\rho_{0}=\frac{\sqrt{1-\chi^{2} w^{2}}}{w^{2}}$. We can also note that the quantum flux $\Phi_{B}$ present in the screw dislocation modifies the energy levels through the change on the eigenvalues of the angular momentum, thus providing us with a relativistic ana$\log$ of the Aharanov-Bohm effect for bound states [1820,48]. Besides, the energy spectrum is a periodic function of the quantum flux $\Phi_{B}$ with periodicity $\Phi_{0}=\frac{2 \pi}{q} \eta$, where $\eta=0,1,2,3, \ldots$, that is, $\mathcal{E}_{k, l, n}\left(\Phi_{B}+\Phi_{0}\right)=\mathcal{E}_{k, l \mp \eta, n}\left(\Phi_{B}\right)$. In addition, the relativistic energy spectrum is influenced by the rotation through the coupling between the eigenvalue of the angular momentum influenced by the Aharonov-Bohm quantum flux $l_{\mathrm{eff}}=l-\frac{q \Phi_{B}}{2 \pi}$ and the angular frequency $w$, giving us, then, a Sagnac-type effect $[22,49,50]$. For $\Phi_{B}=0$ and $\chi \neq 0$ we recover the result obtained in the Ref. [6]; for $\Phi_{B} \neq 0$ and $\chi=0$ we obtain the relativistic energy levels of a charged scalar field subject to the Aharonov-Bohm effect in the Minkowski spacetime in a uniformly rotating frame.

\section{Coulomb-type potential}

The standard procedure of inserting the Coulomb potential into relativistic wave equations is through the minimum coupling $\hat{p}_{\mu} \rightarrow \hat{p}_{\mu}-q A_{\mu}$ via temporal component $A_{0}$ [51]. Another procedure of inserting central potentials is by modifying the mass term of the relativistic wave equations via transformation $m \rightarrow m+V(r)$, where $V(r)$ is a scalar potential. The latter procedure entails a feature which is known in the literature as a position-dependent mass system. This type of system has been studied in atomic physics [52], in the rotating cosmic string spacetime [53,54], on a two-dimensional Klein-Gordon particle [55], quark-antiquark interaction [56] and on a scalar particle in a Gödel-type spacetime [57].

In this section, we insert a Coulomb-type potential into the Klein-Gordon equation by modifying the mass term through 
the transformation

$m \rightarrow m+\frac{a}{\rho}$,

where $a$ is a constant that characterizes the Coulomb-type potential. The Coulomb-type potential has been studied in propagation of gravitational waves [58], in a magnetic quadrupole moment [59], in a neutral particle with permanent magnetic dipole moment [60] and in Lorentz symmetry violation scenarios [61,62]. Then, by substituting the Eq. (15) into the Eq. (6), we obtain from the solution (7) the radial differential equation

$\frac{d^{2} R}{d \rho^{2}}+\frac{1}{\rho} \frac{d R}{d \rho}-\frac{\bar{\iota}^{2}}{\rho^{2}} R-\frac{2 a m}{\rho} R-\bar{\alpha}^{2} R=0$,

where we define the new parameters

$\bar{\iota}^{2}=\left(l-k \chi-\frac{q \Phi_{B}}{2 \pi}\right)^{2}+a^{2} ;$

$\bar{\alpha}^{2}=m^{2}+k^{2}-\left[\mathcal{E}+w\left(l-\frac{q \Phi_{B}}{2 \pi}\right)\right]^{2}$,

where $\bar{\alpha}$ must be real such that we can obtain solutions of bound states.

In order to obtain solutions of bound states, let us consider the case where $a=-|a|$ and the change of variables $\xi=$ $2 \bar{\alpha} \rho$, such that Eq. (17) becomes

$\frac{d^{2} R}{d \xi^{2}}+\frac{1}{\xi} \frac{d R}{d \xi}-\frac{\bar{\iota}^{2}}{\xi^{2}} R+\frac{\beta}{\xi} R-\frac{1}{4} R=0$,

with

$\beta=\frac{|a| m}{\bar{\alpha}}$.

We must now impose that the radial wave function $R(\xi)$ be well behaved at the origin, since it is a singular point of Eq. (18). In this case, for $\lim _{\xi \rightarrow 0} R(\xi)=0$ the solution is $R(\xi) \sim \xi^{|\bar{\imath}|}$. Besides, let us consider the particular case where the frequency of rotation is very small, $w \ll 1$, such that in the vicinity of the fixed point $\rho_{0}=\frac{\sqrt{1-\chi w^{2}}}{w}$ we have $\lim _{w \rightarrow 0} \rho \rightarrow \infty$ which implies $\xi \rightarrow \infty$. In this particular case, for $\lim _{\xi \rightarrow \infty} R(\xi)=0$ the solution is $R(\xi) \sim e^{-\frac{1}{2} \xi}$. Thus, the solution possible for Eq. (18) is given in terms of a function $f(\xi)$ :

$R(\xi)=\xi^{|\bar{\imath}|} e^{-\frac{1}{2} \xi} f(\xi)$.

By substituting the Eq. (20) into the Eq. (18), we have

$\xi \frac{d^{2} f}{d \xi^{2}}+(2|\bar{\imath}|+1-\xi) \frac{d f}{d \xi}+\left(\beta-|\bar{\imath}|-\frac{1}{2}\right) f=0$.

The Eq. (21) is known in the literature as the confluent hypergeometric equation [46] and $f(\xi)$ is a conflu- ent hypergeometric function: $f(\xi)={ }_{1} F_{1}(b, c ; \xi)$, where $b=|\bar{\imath}|+\frac{1}{2}-\beta$ and $c=2|\bar{\imath}|+1$. Again, let us consider the particular case where the angular frequency of rotation is very small, $w \ll 1$ such that in the vicinity of the fixed point $\rho_{0}=\frac{\sqrt{1-\chi^{2} w^{2}}}{w}$ the radial coordinate is very large, that is, $\xi_{0} \rightarrow \infty$. In this particular, for large values of its argument, the confluent hypergeometric function is given by

${ }_{1} F_{1}\left(b, c ; \xi_{0}=2 \bar{\alpha} \rho_{0}\right) \approx \frac{\Gamma(c)}{\Gamma(b)} e^{\xi_{0}} \xi_{0}^{b-c}\left[1+\mathcal{O}\left(\left|\xi_{0}\right|^{-1}\right)\right]$,

where it is diverges for this particular case. Then, in the search for solutions of bound states, it is necessary to impose that $b=-n$, with $n=0,1,2, \ldots$, consequently, the confluent hypergeometric function becomes well behaved for $\xi_{0} \rightarrow \infty$ [46]. Then, with $b=|\bar{\imath}|+\frac{1}{2}-\beta=-n$, we obtain the expression

$$
\begin{aligned}
\mathcal{E}_{k, l, n}= & -w\left(l-\frac{q \Phi_{B}}{2 \pi}\right) \\
& \pm \sqrt{m^{2}+k^{2}-\frac{a^{2} m^{2}}{\left[n+\sqrt{\left(l-k \chi-\frac{q \Phi_{B}}{2 \pi}\right)^{2}+a^{2}}+\frac{1}{2}\right]^{2}}}
\end{aligned}
$$

which represents the relativistic energy levels of a charged scalar field subject to a Coulomb-type potential in a uniformly rotating frame in the spacetime with a magnetic screw dislocation. We can observe the influence of the spacetime topology on the relativistic energy spectrum given in Eq. (23). This influence is characterized by the change in the eigenvalues of the angular moment which are redefined by the parameter $\chi$ and by the quantum flux $\Phi_{B}$, both associated to the topological defect, that is, $l_{\mathrm{eff}}=l-k \chi-\frac{q \Phi_{B}}{2 \pi}$, resulting in an effect analogous to the Aharanov-Bohm effect for bound states [30]. We can also note that the relativistic energy spectrum is a periodic function of the quantum flux $\Phi_{B}$ with periodicity $\Phi_{0}=\frac{2 \pi}{q} \eta$, that is, $\mathcal{E}_{k, l, n}\left(\Phi_{B}+\Phi_{0}\right)=\mathcal{E}_{k, l \mp \eta, n}\left(\Phi_{B}\right)$, where $\eta=0,1,2, \ldots$. Again, we can also observe a Sagnactype effect $[22,49,50]$, which is characterized by the coupling between the angular frequency $w$ and the effective angular momentum quantum number $l_{\text {eff }}=l-\frac{q \Phi_{B}}{2 \pi}$. By making $w=0$ we recover the result obtained in the Ref. [19]. For $\Phi_{B}=0$ and $\chi \neq 0$, the Eq. (23) represents the relativistic energy levels of scalar field of position-dependent mass under effects of a Coulomb-type potential in a uniformly rotating frame in the spacetime with a screw dislocation; for $\Phi_{B} \neq 0$ and $\chi=0$, the Eq. (23) represents the relativistic energy levels of charged scalar field of position-dependent mass under effects of a Coulomb-type potential in the Minkowski spacetime. 


\section{Klein-Gordon oscillator}

Based on the model for a relativistic quantum oscillator that interacts with a spin- $\frac{1}{2}$ fermionic field, which is known in the literature as the Dirac oscillator [63-74], Bruce and Minning proposed a model for a relativistic quantum oscillator that interacts with a scalar field, where this model has become known in the literature as the Klein-Gordon oscillator [31,75-86]. The Klein-Gordon oscillator is described through the coupling $\partial_{\mu}+m \omega X_{\mu}$ into the Klein-Gordon equation, where $\omega$ is the angular frequency of the KleinGordon oscillator and $X_{\mu}=(0, \rho, 0,0)$. In this way, the Eq. (4) is rewritten in the form $[17,19]$ :

$$
\begin{aligned}
& \frac{1}{\sqrt{-g}}\left(\partial_{\mu}-i q A_{\mu}+m \omega X_{\mu}\right)\left(\sqrt{-g} g^{\mu v}\right) \\
& \times\left(\partial_{v}-i q A_{v}-m \omega X_{v}\right) \phi-m^{2} \phi=0 .
\end{aligned}
$$

In the spacetime with a magnetic screw dislocation described by the line element (2) and the 4-vector potential (5), and following the steps from Eq. (6) to the Eq. (8), we obtain the following radial equation

$\frac{d^{2} R}{d \rho^{2}}+\frac{1}{\rho} \frac{d R}{d \rho}-\frac{\iota^{2}}{\rho^{2}} R-m^{2} \omega^{2} \rho^{2} R+\gamma R=0$,

where $\iota$ is defined in the Eq. (9) and

$\gamma=\left[\mathcal{E}+w\left(l-\frac{q \Phi_{B}}{2 \pi}\right)\right]^{2}-m^{2}-k^{2}-2 m \omega$.

Let us consider the change of variables $\varrho=m \omega \rho^{2}$, such that Eq. (27) becomes

$\frac{d^{2} R}{d \varrho^{2}}+\frac{1}{\varrho} \frac{d R}{d \varrho}-\frac{\iota^{2}}{4 \varrho^{2}} R+\frac{\gamma}{4 m \omega \varrho} R-\frac{1}{4} R=0$.

Again, we must now impose that the radial wave function $R(\xi)$ be well behaved. In this case, for $\lim _{\xi \rightarrow 0} R(\xi)=0$ the solution is $R(\xi) \sim \xi^{\frac{|\iota|}{2}}$. Let us consider the particular case where the frequency of rotation is very small, $w \ll 1$, such that in the vicinity of the fixed point $\rho_{0}=\frac{\sqrt{1-\chi w^{2}}}{w}$ we have $\lim _{w \rightarrow 0} \rho \rightarrow \infty$ which implies $\varrho \rightarrow \infty$. In this particular case, for $\lim _{\varrho \rightarrow \infty} R(\varrho)=0$ the solution is $R(\varrho) \sim e^{-\frac{1}{2} \varrho}$. Thus, the solution for the Eq. (27) is given in terms of a function $g(\varrho)$ :

$R(\varrho)=\varrho^{\frac{|\iota|}{2}} e^{-\frac{1}{2} \varrho} g(\varrho)$.

Then, by substituting the Eq. (28) into the Eq. (27), we obtain

$\varrho \frac{d^{2} g}{d \varrho^{2}}+(|\iota|+1-\varrho) \frac{d g}{d \varrho}-\left(\frac{|\iota|}{2}+\frac{1}{2}-\frac{\gamma}{4 m \omega}\right) g=0$.

Note that the Eq. (29) is also the confluent hypergeometric equation [46] and $g(\varrho)$ is the confluent hypergeometric function: $g(\varrho)={ }_{1} F_{1}(\bar{b}, \bar{c} ; \varrho)$, where $\bar{b}=\frac{|\iota|}{2}+\frac{1}{2}-\frac{\gamma}{4 m \omega}$ and $\bar{c}=|\iota|+1$.

It is possible to discuss two energy profiles for this system. One for any value of the angular frequency of rotation which induces a hard-wall potential (general case) and another for very small values of the angular frequency of rotation (particular case).

\subsection{General case}

For an arbitrary value of the angular frequency of rotation implies in a similar case seen in Sect. 2, that is, the wave function must vanish in $\varrho_{0}=\frac{m \omega\left(1-\chi^{2} w^{2}\right)}{w^{2}}$, restriction imposed by the rotation. This means that the charged scalar field is restricted in a region where this restriction is characterized by the presence of a hard-wall potential induced by the effects of rotation in a spacetime with a magnetic screw dislocation. This kind of confinement is described by the boundary condition given in the Eq. (12).

Let us consider the case where $\frac{\gamma}{4 m \omega} \gg 1$, or $m \omega \ll 1$, while $\bar{c}$ is fixed. In this case, the parameter $\bar{b}$ can be considered large and, in the fixed point $\varrho_{0},{ }_{1} F_{1}(\bar{b}, \bar{c} ; \varrho)$ can be written in the form [47]:

$$
\begin{aligned}
g\left(\varrho_{0}\right) & ={ }_{1} F_{1}\left(\bar{b}, \bar{c} ; \varrho_{0}\right) \\
& \propto \cos \left(\frac{\pi}{4}-\frac{\bar{c}}{2}+\sqrt{2(\bar{c}-2 \bar{b}) \varrho_{0}}\right) .
\end{aligned}
$$

Thus, by substituting the Eqs. (28) and (30) into the Eq. (12), we obtain expression

$$
\begin{aligned}
& \mathcal{E}_{k, l, n} \approx-w\left(l-\frac{q \Phi_{B}}{2 \pi}\right) \\
& \pm \sqrt{m^{2}+k^{2}+2 m \omega+\frac{\pi^{2} w^{2}}{\left(1-\chi^{2} w^{2}\right)}\left(n+\frac{1}{2}\left|l-k \chi-\frac{q \Phi_{B}}{2 \pi}\right|+\frac{3}{4}\right)^{2}},
\end{aligned}
$$

which represents the relativistic energy spectrum of a charged scalar field subject to a Klein-Gordon oscillator and a hardwall potential induced by a uniformly rotating frame in the spacetime with a magnetic screw dislocation. We can see that the relativistic energy spectrum (31) is influenced by the spacetime topology through the redefinition of the eigenvalues of the angular momentum in terms of the parameters $\chi$ and $\Phi_{B}$, both associated to the magnetic screw dislocation, $l_{\mathrm{eff}}=l-k \chi-\frac{q \Phi_{B}}{2 \pi}$, producing an effect analogous to the Aharonov-Bohm effect for bound states [30]. We can also note that the relativistic energy spectrum is a periodic function of the quantum flux $\Phi_{B}$ with periodicity $\Phi_{0}=\frac{2 \pi}{q} \eta$, where $\eta=0,1,2, \ldots$, that is, $\mathcal{E}_{k, l, n}\left(\Phi_{B}+\Phi_{0}\right)=$ $\mathcal{E}_{k, l \mp \eta, n}\left(\Phi_{B}\right)$. Again, we can observe a Sagnac-type effect $[22,49,50]$ on relativistic energy levels from the rotating effects, which is influenced by the quantum flux of the topological defect. In contrast to the Eq. (14), we have a new 
contribution to the relativistic energy spectrum given by the term $2 m \omega$ that stems from the Klein-Gordon oscillator. For $\Phi_{B}=0$ and $\chi \neq 0$ we have relativistic energy levels of a scalar field subject to the Klein-Gordon oscillator and a hard-wall potential induced by a uniformly rotating frame in the spacetime with a screw dislocation; for $\Phi_{B} \neq 0$ and $\chi=0$ we have relativistic energy levels of a charged scalar field subject to the Klein-Gordon oscillator and a hard-wall potential induced by a uniformly rotating frame subject to the Aharonov-Bohm effect in the Minkowski spacetime.

\subsection{Particular case}

Now, let us consider the particular case in which the frequency of rotation is very small, $w \ll 1$ so that the radial coordinate in the vicinity of the point fixed $\varrho_{0}$ tends to the infinity, as seen in Sect. 3. In this particular case, the confluent hypergeometric function admits polynomial solutions of degree $n$ imposing that $\bar{b}=-n$, with $n=0,1,2, \ldots$ Then, by following the discussion made in the Eq. (22) to the (23), we obtain the expression

$$
\begin{aligned}
\mathcal{E}_{k, l, n}= & -w\left(l-\frac{q \Phi_{B}}{2 \pi}\right) \\
& \pm \sqrt{m^{2}+k^{2}+4 m \omega\left(n+\frac{1}{2}\left|l-k \chi-\frac{q \Phi_{B}}{2 \pi}\right|+1\right)}
\end{aligned}
$$

which represents the relativistic energy levels of a charged scalar field subject to the Klein-Gordon oscillator in a uniformly rotating frame in the spacetime with a magnetic screw dislocation. We can note that the relativistic energy levels (31) is influenced by the spacetime topology through the redefinition of the eigenvalues of the angular momentum in terms of the parameters $\chi$ and $\Phi_{B}$, both associated to the topological defect, $l_{\text {eff }}=l-k \chi-\frac{q \Phi_{B}}{2 \pi}$, producing an relativistic effect analogous to the Aharonov-Bohm effect for bound states [30]. We can also note that the relativistic energy spectrum is a periodic function of the quantum flux $\Phi_{B}$ with periodicity $\Phi_{0}=\frac{2 \pi}{q} \eta$, where $\eta=0,1,2, \ldots$, that is, $\mathcal{E}_{k, l, n}\left(\Phi_{B}+\Phi_{0}\right)=\mathcal{E}_{k, l \mp \eta, n}\left(\Phi_{B}\right)$. In addition, again, we can see a Sagnac-type effect $[22,49,50]$ on relativistic energy levels from the rotating effects, which is influenced by the quantum flux of the magnetic screw dislocation. By taking $w=0$ we recover the particular results obtained in the Refs. $[17,18]$. For $\Phi_{B}=0$ and $\chi \neq 0$ we have relativistic energy levels of a scalar field subject to the Klein-Gordon oscillator in a uniformly rotating frame in the spacetime with a screw dislocation; for $\Phi_{B} \neq 0$ and $\chi=0$ we have relativistic energy levels of a charged scalar field subject to the KleinGordon oscillator in a uniformly rotating frame subject to the Aharonov-Bohm effect in the Minkowski spacetime; for
$\Phi_{B}=\chi=0$ we recover the result obtained in the Ref. [26] without cosmic string.

\section{Conclusion}

We have investigated the effects of a uniformly rotating frame on a charged scalar field in the spacetime with a magnetic screw dislocation. Due to the rotating effects in this background, we can note that the radial coordinate is restricted and this restriction is determined by the spacetime topology. Through this restriction in the radial coordinate, we determine solutions of bound states, hence, we extract the energy profiles for the systems analyzed. Our investigation starts with the hard-wall potential, which, from the mathematical point of view, is a Dirichlet boundary condition imposed by the rotating effects, and with that we determine the relativistic energy profile of this system.

Through the definition of position-dependent mass system, we inserted a Coulomb-type potential into the KleinGordon equation by modifying the mass term. Analytically, for very small values of the frequency of rotation, we determine solutions of bound states and we can note that the presence of the Coulomb-type potential modifies the energy spectrum of the system.

We also investigate the Klein-Gordon oscillator, where, for well-defined rotating frequency scales, it is possible to determine two energetic profiles for this system. First, we determine the energy profile of the Klein-Gordon oscillator in a uniformly rotating frame for arbitrary values of the frequency of rotation, which induces a hard-wall potential. Next for very small values of the frequency of rotation. We can see that the two energy profiles are totally different.

It is worth mentioning that in all cases we can note the influence of the spacetime topology by redefining the angular momentum eigenvalue which is described in terms of the parameter associated with the screw dislocation and the parameter associated with the internal quantum flux of the defect. Consequently, the Sagnac-type effect, which arises at all energy levels due to rotation, is also influenced by the internal quantum flux of the topological defect.

Acknowledgements The author would like to thank CNPq (Conselho Nacional de Desenvolvimento Científico e Tecnológico, Brazil). Ricardo L. L. Vitória was supported by the $\mathrm{CNPq}$ project No. 150538/2018-9.

Data Availability Statement This manuscript has no associated data or the data will not be deposited. [Authors' comment: This is a theoretical study and no experimental data has been listed.]

Open Access This article is distributed under the terms of the Creative Commons Attribution 4.0 International License (http://creativecomm ons.org/licenses/by/4.0/), which permits unrestricted use, distribution, and reproduction in any medium, provided you give appropriate credit 
to the original author(s) and the source, provide a link to the Creative Commons license, and indicate if changes were made. Funded by SCOAP ${ }^{3}$.

\section{References}

1. M.O. Katanaev, I.V. Volovich, Ann. Phys. 216, 1 (1992)

2. R.A. Puntigam, H.H. Soleng, Class. Quantum Gravity 14, 1129 (1997)

3. K.C. Valanis, V.P. Panoskaltsis, Acta Mech. 175, 77 (2005)

4. H. Kleinert, Gauge Fields in Condensed Matter, vol. 2 (World Scientific, Singapore, 1989)

5. A.V.D.M. Maia, K. Bakke, Phys. B 531, 213 (2018)

6. R.L.L. Vitória, K. Bakke, Eur. Phys. J. C 78, 175 (2018)

7. C. Furtado, F. Moraes, J. Phys. A Math. Gen. 33, 5513 (2000)

8. M.J. Bueno, C. Furtado, K. Bakke, Phys. B Condens. Matter 496, 45 (2016)

9. A.L. Silva Netto, C. Furtado, J. Phys. Condens. Matter 20, 125209 (2008)

10. C. Furtado, F. Moraes, Europhys. Lett. 45(3), 279 (1999)

11. G.A. Marques, C. Furtado, V.B. Bezerra, F. Moraes, J. Phys. A Math. Gen. 34, 5945 (2001)

12. K. Bakke, Phys. B Condens. Matter 537, 346 (2018)

13. K. Bakke, L.R. Ribeiro, C. Furtado, Cent. Eur. J. Phys. 8(6), 893 (2010)

14. K. Bakke, Ann. Phys. (NY) 346, 51 (2014)

15. J. Carvalho, C. Furtado, F. Moraes, Phys. Rev. A 84, 032109 (2011)

16. K. Bakke, C. Furtado, Ann. Phys. (NY) 336, 489 (2013)

17. J. Carvalho, A.M. de M. Carvalho, E. Cavalcante, C. Furtado, Eur. Phys. J. C 76, 365 (2016)

18. R.L.L. Vitória, K. Bakke, Int. J. Mod. Phys. D 27, 1850005 (2018)

19. R.L.L. Vitória, K. Bakke, Eur. Phys. J. Plus 133, 490 (2018)

20. R.L.L. Vitória, K. Bakke, Gen. Relativ. Gravit. 48, 161 (2016)

21. L.D. Landau, E.M. Lifshitz, The Classical Theory of Fields, Course of Theoretical Physics, vol. 2, 4th edn. (Elsevier, Oxford, 1980)

22. F.W. Hehl, W.-T. Ni, Phys. Rev. D 42, 2045 (1990)

23. K. Bakke, Ann. Phys. (Berlin) 523, 762 (2011)

24. P. Strange, L.H. Ryder, Phys. Lett. A 380, 3465 (2016)

25. L.B. Castro, Eur. Phys. J. C 76, 61 (2016)

26. L.C.N. Santos, C.C. Barros Jr., Eur. Phys. J. C 78, 13 (2018)

27. M. Hosseinpour, H. Hassanabadi, Eur. Phys. J. Plus 130, 236 (2015)

28. H.F. Mota, K. Bakke, Gen. Relativ. Gravit. 49, 104 (2017)

29. Y. Aharonov, D. Bohm, Phys. Rev. 115, 485 (1959)

30. M. Peshkin, A. Tonomura, The Aharonov-Bohm Effect in Lecture Notes in Physics, vol. 340 (Springer, Berlin, 1989)

31. S. Bruce, P. Minning, Nuovo Cimento A 106, 711 (1993)

32. E.R. Bezerra de Mello, Braz. J. Phys. 31, 211 (2001)

33. A.L. Cavalcanti de Oliveira, E.R. Bezerra de Mello, Class. Quantum Gravity 23, 5249 (2006)

34. A. Boumali, H. Aounallah, Adv. High Energy Phys. 2018, 1031763 (2018)

35. R. Jackiw, A.I. Milstein, S.-Y. Pi, I.S. Terekhov, Phys. Rev. B 80, 033413 (2009)

36. M.A. Anacleto, I.G. Salako, F.A. Brito, E. Passos, Phys. Rev. D 92 , $125010(2015)$

37. V.R. Khalilov, Eur. Phys. J. C 74, 2708 (2014)

38. C. Coste, F. Lund, M. Umeki, Phys. Rev. E 60, 4908 (1999)

39. C. Furtado, F. Moraes, V. Bezerra, Phys. Rev. D 59, 107504 (1999)

40. E.V.B. Leite, K. Bakke, H. Belich, Adv. High Energy Phys. 2015, $925846(2015)$

41. H. Belich, E.O. Silva, M.M. Ferreira Jr., M.T.D. Orlando, Phys. Rev. D 83, 125025 (2011)
42. K. Bakke, Int. J. Theor. Phys. 54, 2119 (2015)

43. K. Bakke, H. Belich, J. Phys. G Nucl. Part. Phys. 42, 095001 (2015)

44. K. Bakke, C. Furtado, Eur. Phys. J. B 87, 222 (2014)

45. K. Bakke, Eur. Phys. J. B 85, 354 (2012)

46. G.B. Arfken, H.J. Weber, Mathematical Methods for Physicists, 6th edn. (Elsevier Academic Press, New York, 2005)

47. M. Abramowitz, I.A. Stegum, Handbook of Mathematical Functions (Dover Publications Inc., New York, 1965)

48. V.B. Bezerra, J. Math. Phys. 38, 2553 (1997)

49. M.G. Sagnac, C. R. Acad. Sci. (Paris) 157, 708 (1913)

50. M.G. Sagnac, C. R. Acad. Sci. (Paris) 157, 1410 (1913)

51. W. Greiner, Relativistic Quantum Mechanics: Wave Equations, 3rd edn. (Springer, Berlin, 2000)

52. G. Soff, B. Müller, J. Rafelski, W. Greiner, Z. Naturforsch. 28a, 1389 (1973)

53. M.S. Cunha, C.R. Muniz, H.R. Christiansen, V.B. Bezerra, Eur. Phys. J. C 76, 512 (2016)

54. Z. Wang, Z. Long, C. Long, B. Wang, Can. J. Phys. 95(4), 331 (2017)

55. S.M. Ikhdaira, M. Hamzavi, Chin. Phys. B 21, 110302 (2012)

56. M.K. Bahar, F. Yasuk, Adv. High Energy Phys. 2013, 814985 (2013)

57. R.L.L. Vitória, C. Furtado, K. Bakke, Eur. Phys. J. C 78, 44 (2018)

58. H. Asada, T. Futamase, Phys. Rev. D 56, R6062 (1997)

59. I.C. Fonseca, K. Bakke, J. Math. Phys. 56, 062107 (2014)

60. P.M.T. Barboza, K. Bakke, Ann. Phys. 361, 259 (2015)

61. K. Bakke, H. Belich, Ann. Phys. 360, 596 (2015)

62. R.L.L. Vitória, H. Belich, K. Bakke, Adv. High Energy Phys. 2017, 6893084 (2017)

63. M. Moshinsky, A. Szczepaniak, J. Phys. A Math. Gen. 22, L817 (1989)

64. Y. Nogami, F.M. Toyama, Can. J. Phys. 74, 114 (1996)

65. W. Moreau, R. Easther, R. Neutze, Am. J. Phys. 62, 531 (1994)

66. V.M. Villalba, Eur. J. Phys. 15, 191 (1994)

67. N.A. Rao, B.A. Kagali, Mod. Phys. Lett. A 19, 2147 (2004)

68. Victor M. Villalba, Phys. Rev. A 49, 1 (1994)

69. J. Cravalho, C. Furtado, F. Moraes, Phys. Rev. A 84, 032109 (2011)

70. K. Bakke, C. Furtado, Ann. Phys. 336, 489 (2013)

71. K. Bakke, H.F. Mota, Eur. Phys. J. Plus 133, 409 (2018)

72. L. Deng, C. Long, Z. Long, Ting Xu, Adv. High Energy Phys. 2018, 2741694 (2018)

73. A. Boumali, H. Hassanabadi, Eur. Phys. J. Plus 128, 124 (2013)

74. H. Hassanabadi, S. Sargolzaeipor, B.H. Yazarloo, Few-Body Syst. 56, 115 (2015)

75. N.A. Rao, B.A. Kagali, Phys. Scr. 77, 015003 (2008)

76. J.-Y. Cheng, Int. J. Theor. Phys. 50, 228 (2011)

77. B. Mirza, R. Narimani, S. Zare, Commun. Theor. Phys. 55, 405 (2011)

78. M.-L. Liang, R.-L. Yang, Int. J. Mod. Phys. A 27, 1250047 (2012)

79. A. Boumali, N. Messai, Can. J. Phys. 92, 11 (2014)

80. K. Bakke, C. Furtado, Ann. Phys. (NY) 355, 48 (2015)

81. R.L.L. Vitória, K. Bakke, Eur. Phys. J. Plus 131, 36 (2016)

82. R.L.L. Vitória, C. Furtado, K. Bakke, Ann. Phys. (NY) 370, 128 (2016)

83. R.L.L. Vitória, H. Belich, K. Bakke, Eur. Phys. J. Plus 132, 25 (2017)

84. R.L.L. Vitória, H. Belich, Eur. Phys. J. C 78, 999 (2018)

85. B. Khosropour, Indian J. Phys. 92(1), 43 (2018)

86. B. Hamil, M. Merad, Eur. Phys. J. Plus 133, 174 (2018) 